\title{
An Experiment for Two-Color Photoionization using High Intensity Extreme-UV Free Electron and Near-IR Laser Pulses
}

P. Radcliffe ${ }^{\text {a }}$, S. Düsterer ${ }^{\text {a,* }}$, A. Azima ${ }^{a}$, W. B. Li ${ }^{\text {a }}$, E. Plönjes ${ }^{\mathrm{a}}$, H. Redlin ${ }^{\mathrm{a}}$, J. Feldhaus ${ }^{\mathrm{a}}$, P. Nicolosi ${ }^{\mathrm{b}}$, L. Poletto ${ }^{b}$, J. Dardis ${ }^{c}$, J. P. Gutierrez ${ }^{c}$, P. Hough ${ }^{c}$, K. D. Kavanagh ${ }^{c}$, E. T. Kennedy ${ }^{\text {c }}$, H. Luna ${ }^{\text {c }}$, P. Yeates ${ }^{\text {c }}$, J. T. Costello ${ }^{c}$, A. Delyseries ${ }^{\mathrm{d}}$, C. L. S. Lewis ${ }^{\mathrm{d}}$, D. Glijer ${ }^{\mathrm{e}}$, D. Cubaynes ${ }^{\mathrm{e}}, \mathrm{M}$. Meyer ${ }^{\mathrm{e}}$

${ }^{a}$ Hamburger Synchrotronstrahlungslabor HASYLAB at Deutsches Elektronen-Synchrotron DESY, Notkestr. 85, D-22607 Hamburg, Germany

${ }^{\mathrm{b}}$ INFM-LUXOR, Department of Information Engineering, University of Padova, Via Gradenigo 6/A, 35131 Padova, Italy

c National Center for Plasma Science and Technology, Dublin City University, Dublin, Ireland and School of Physical Sciences, Dublin City University, Dublin 9, Ireland

${ }^{\mathrm{d}}$ International Research Centre for Experimental Physics, Queens University Belfast BTry 1NN Northern Ireland, United Kingdom e LIXAM/CNRS, UMR 8624 Centre Universitaire Paris-Sud, Bâtiment 350, 


\begin{abstract}
1 We describe an experimental system designed for single-shot photoelectron spec2 troscopy on free atoms and molecules at the Free Electron Laser in Hamburg 3 (FLASH at DESY). The combination of the extreme ultra-violet (EUV) Free Elec4 tron Laser and a temporally synchronized optical fs laser (Ti:Sapphire) enables a 5 variety of two-color pump-probe experiments. The spectral, temporal and spatial 6 characteristics of both the EUV FEL and the optical laser pulses, the experimen7 tal procedure to control their overlap as well as the performance of an electron 8 spectrometer used to obtain single-shot photoelectron spectra are discussed. As an 9 illustration of the capabilities of this set-up, some results on two-photon two-color 10 ionization of rare gases are presented.
\end{abstract}

Key words: Photoionization, photoelectron spectroscopy, free electron lasers, magnetic bottle spectrometer, EUV spectroscopy, femtosecond lasers, two color photoionization

\title{
1 INTRODUCTION
}

The operation of FLASH, beginning in mid-2005 has opened up many fascinating possibilities for new experimental studies, which make use of ultra-short laser pulses at high fundamental photon energies $(13-48 \mathrm{~nm})$ with high peak and average power $[1,2]$. A complementary optical laser facility has been set up at FLASH which provides femtosecond infra-red (110 fs, $800 \mathrm{~nm}$ ) and picosec-

* Corresponding author. Tel.: +494089981625; fax: +494089984475

Email address: Stefan.Duesterer@desy.de (S. Düsterer). 
ond visible (12 ps, $523 \mathrm{~nm}$ ) pulses, electronically synchronized with the Free Electron Laser (pulse duration 10 - $50 \mathrm{fs}$ ) pulses. Pump-probe experiments and time-resolved studies with intense EUV + near-infrared/optical fields on the femtosecond time scale become feasible and will nicely extend present EUV +NIR/Vis investigations at synchrotron radiation facilities, which focus on spectroscopic features in the photoexcitation and photoionization of atoms (e.g. [3] and references therein) and molecules (e.g. [4,5,6]). Using the radiation from FLASH the dynamics of molecular fragmentation can be investigated by combining the radiation of two distinctly different wavelengths, one in the EUV, and the other in the near-infrared/optical regime. To take best advantage of the independent sources, the synchronization and the spatial and temporal stability of the set-up has to be characterized under real experimental conditions.

Above threshold ionization (ATI) is a powerful tool to obtain information about the temporal overlap of femtosecond pulses. Briefly, this technique uses photoelectron spectroscopy of rare gas atoms to analyze the photoionization signal produced by an EUV photon in the presence of a strong optical laser beam. The intense optical field $\left(>10^{10} \mathrm{Wcm}^{-2}\right)$ dresses the photo-ejected electrons, in other words, the electrons are born into the optical field where they can emit or absorb one or more additional light quanta, which are observed as a sideband structure on both sides of the main atomic line in the photoelectron spectrum $[7,8]$. The intensity and shape of the sidebands depend heavily on the temporal and spatial profiles of the two femtosecond pulses [8] and can therefore be utilized to characterize the EUV pulses $[9,10]$, and to measure directly the time delay between both laser pulses [11].

The main motivation for the present paper is to describe the ultrafast and in- 
tense two-color photoionization facility at FLASH and to present and illustrate its capabilities. An important aim is also to provide guidance on practical experimental design considerations that arise. The paper is organized as follows. In Section 2, we briefly describe the current status of the FLASH facility and the synchronized optical laser system. The different FLASH beam diagnostic tools are summarized, in particular those specifically added for our experiments. We include recent results on the harmonic content in the EUV beam. In Section 3, we describe the single-shot performance of the magnetic bottle spectrometer used to record photoelectron spectra as well as the imaging technique used to ensure the spatial overlap of the FLASH and femtosecond optical laser pulses. We also outline the main characteristics of the fast photodiode and streak camera systems used to monitor and measure the time delay between FLASH and the optical laser beams. In Section 4, we present some examples demonstrating how the present set-up can be used to study two-color photoionization processes in rare gases. Finally we draw some conclusions and point to planned experimental developments.

\section{FLASH PUMP-PROBE FACILITY AND DIAGNOSTICS}

\subsection{EUV Free Electron Laser}

FLASH has been described in some detail in a number of papers [1,2] (and references within) and so we confine this section to a brief update. In its current mode of operation the EUV-FEL FLASH is operated in either singlebunch mode at repetition rate of $5 \mathrm{~Hz}$ or in multibunch mode with up to 30 bunches, separated from one another by 1,4 , or $10 \mu$ s depending on the 
users requirements. The fundamental wavelengths have so far ranged from 13 - $48 \mathrm{~nm}$. FLASH reached saturation producing average energies of ca. $70 \mu \mathrm{J}$ and maximum values of more than $170 \mu \mathrm{J}[2]$.

In order to fully characterize the performance of FLASH at DESY, a large variety of photon diagnostic tools have been developed over several years, for further details see Refs. $[12,13,14,15]$. The FEL pulse energies are measured using a gas monitor detector (GMD), covering a dynamic range of about seven orders of magnitude with an absolute energy resolution of $\sim 10 \%$ [16]. FLASH has several beamlines for user experiments, see Fig. 1. Three utilize the FEL beam with the full spectral bandwidth $(0.5-1 \%)$ while having different focussing conditions: BL1: $100 \mu \mathrm{m}$ full width half maximum FWHM, BL2: $15-25 \mu \mathrm{m}$ FWHM [17] and BL3 unfocussed. On the other branch, the fourth beamline (PG2) consists of a high resolution monochromator for spectroscopic experiments with a nominal focus of $100 \mu \mathrm{m}$ [18].

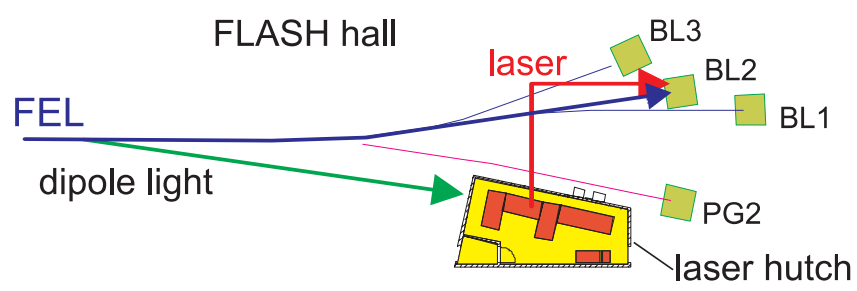

Fig. 1. Layout of the FLASH experimental hall with the different experimental stations and the safety hutch for the optical laser.

It is well known that FEL beams comprise not just the fundamental but also higher harmonics $[15,19]$. Hence it is important to be able to measure the spectral composition of the beam. At this time FLASH has a grazing incidence spectrometer system located in the tunnel near the exit aperture of the final undulator section [19]. Briefly, the system contains a spherical variable line spacing grating, which provides a flat field in the focal plane for 
easy matching to a flat detector array. Its spectral resolution is better than 1500 across its $3-48 \mathrm{~nm}$ operating wavelength range. Hence it was possible to measure spectra of the fundamental (Fig. 2) as well as the second and third harmonic FEL pulses [15]. The detection system consists of a phosphor faceplate coupled to an intensified CCD camera. With its gated readout, it is suitable for selecting single pulses from a FEL pulse train [2]. To obtain spectra of the second and third harmonics with this system, it is necessary to integrate many tens of shots. However, this set-up uses the direct FLASH beam, i.e. the FEL pulses can not be used for other experiments at the same time. In order to obtain single-shot harmonic spectra in a parasitic manner we built a flat field spectrometer system $(\omega-5 \omega[2])$, with back illuminated CCD readout, in tandem with the main experimental chamber, see Fig. 7.

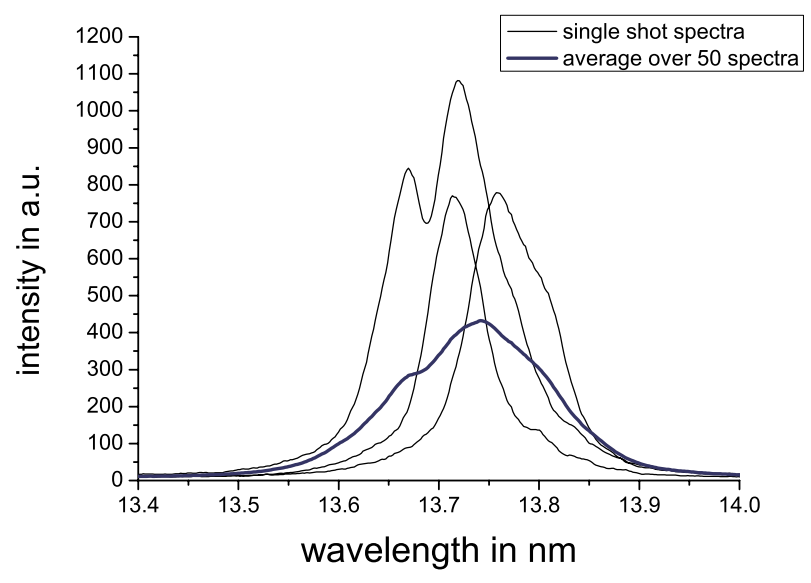

Fig. 2. Spectral distribution of individual pulses from FLASH lasing at $13.7 \mathrm{~nm}$. The spectra were recorded with the grazing incidence monochromator located in the tunnel. The spectrum integrated over many pulses is given as solid black line.

The system was equipped with a Harada type variable line spaced grating [20]. The spectral image was recorded on a sensitive back illuminated CCD built by Andor Technology of Belfast, Northern Ireland which comprised $2048 \times$ 
512 pixels of dimension $13 \mu \mathrm{m} \times 13 \mu \mathrm{m}$ each. A 1200 grooves $/ \mathrm{mm}$ grating was selected since it permitted us to observe the fundamental, second and third harmonics of the FEL on a single CCD image at the wavelength of $13.7 \mathrm{~nm}$ that we selected for our experiments. The spectrometer was operated without an entrance slit, which made alignment relatively easy while ensuring high flux throughput. It also afforded a degree of spatial resolution in the direction orthogonal to the wavelength dispersion axis. The associated compromise in spectral resolution, which was reduced to approximately $\sim 100$ in this mode, did not pose a problem as the harmonics are well separated in wavelength. One feature of the Harada type grating is its high efficiency in high spectral orders. This can be both a blessing and a curse. The former, since it permits one to record high order FEL harmonics in high spectral orders thereby obviating the need to move the CCD during an experiment. The latter, since this gives rise to unwanted overlapping of these spectral features, e.g., the $\mathrm{N}^{\text {th }}$ spectral order of harmonic-N will overlap the fundamental. For this reason, we used a range of thin film EUV filters (mainly Polypropylene and Aluminium of thicknesses from $1-4 \mu \mathrm{m}$ ) to enable order sorting. In Figure 3 (top panel) we show a 3D presentation with a series of single-shot raw spectra obtained with the flat field spectrometer. A corresponding single pulse spectrum is given in the bottom panel of Figure 3.

The FEL was operated at a fundamental wavelength of $13.7 \mathrm{~nm}$. The spectra show the fundamental, second $(2 \omega)$ and third $(3 \omega)$ harmonics of the FLASH pulses in first and second order of the diffraction grating. We used a polythene (CH) filter which provided high transmission for the second and third harmonics of the FEL (see transmission on inset). The filter had the beneficial effect of suppressing higher order harmonics so that the third and second harmonic 

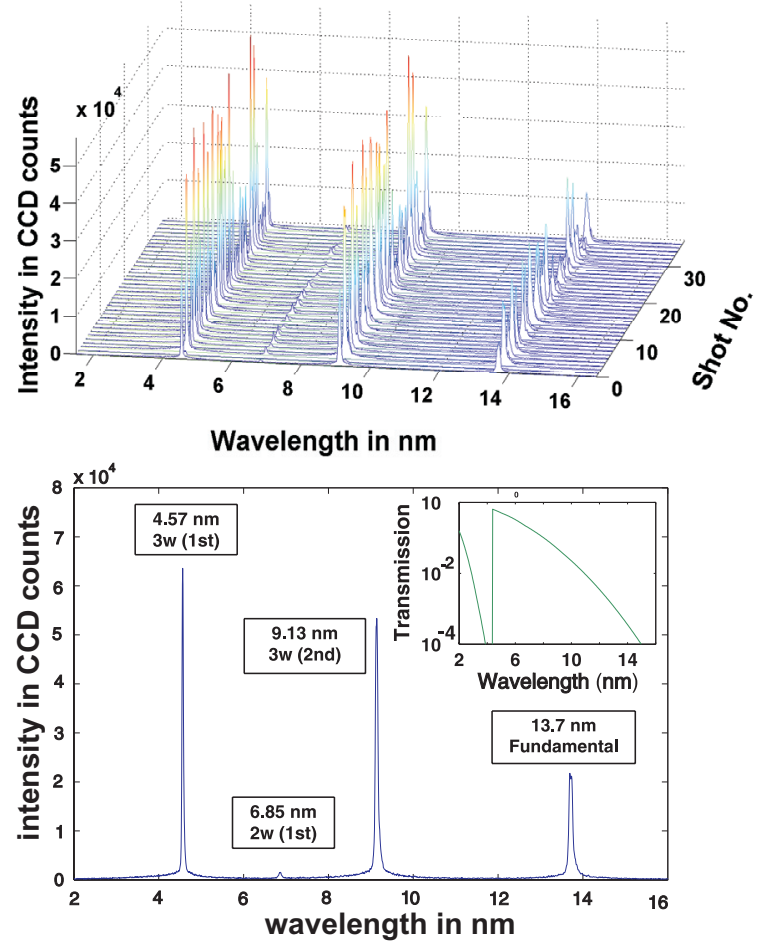

Fig. 3. Spectral distribution of the FLASH beam lasing at $13.7 \mathrm{~nm}$. (Top) Series of single shot spectra. (Bottom) Raw CCD data for one single FLASH pulse. (inset) Transmission of the polythene filter used to optimize the transmission of the second and third harmonics. 
features were free of any order sorting problems. It is clear that the harmonic pulse energies vary from shot to shot as expected from a source based on the principle of SASE (Self Amplified Spontaneous Emission) where the radiation field builds up from shot noise in the undulator.

During our photoelectron measurements in summer 2005, we observed a high second harmonic content in the FEL beam $(0.5 \%)[15,19]$, comparable to the third harmonic, indicating strong fluctuation of the electron beam normal to the undulator direction. A significant second harmonic radiation content in the FEL beam can be a problem as it can mask two photon processes, which are one of the main research directions mapped our for FLASH. In Figure 4 we show a spectrum in the wavelength region of the second and third harmonics in the first (diffraction) order of the grating. The spectrum has been corrected for filter transmission as well as grating and CCD efficiencies.

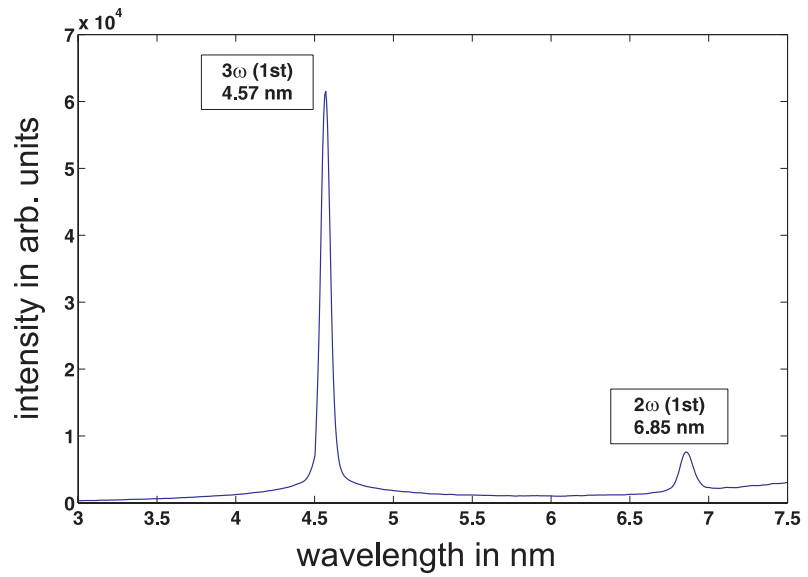

Fig. 4. Low wavelength part of the spectral distribution of FLASH lasing at $13.7 \mathrm{~nm}$. The spectrum has been corrected for the wavelength dependent transmission of the grating and the filters as well as for the CCD efficiencies.

These measurements show that the ratio of the second to third harmonic pulse energies has dropped dramatically since 2005 (1:8 compared to 1:1 in 2005). The data shown here were recorded in August of 2006 when FLASH reached 
saturated operation and record EUV pulse energies [2]. The result demonstrates nicely how continuous development of FLASH over the intervening period resulted in near ideal operation.

\subsection{Pulsed optical laser}

The other key element of the two-color facility is the ultrafast optical laser system. The important figures of merit for an optical laser system is the ability to deliver to the experimental endstations ultrashort pulses with the time structure of the FEL itself [21]. In short, the laser comprises three modular sub-systems: The first one, the pump laser, is a slightly modified copy of the photocathode laser of the FEL [22]. This Nd:YLF based burst mode laser was optimized to produce pulse trains of up to 800 pulses with a spacing of $1 \mu \mathrm{s}$ between the individual laser pulses. The pulse trains can be produced with 2 or $5 \mathrm{~Hz}$. Following frequency doubling, green laser pulses $(\lambda=523 \mathrm{~nm})$ with energies of up to $0.4 \mathrm{~mJ}$ and a pulse duration of $12 \mathrm{ps}$ can be obtained. A second laser (Ti:Sapphire) is used to produce ultra-short $(\sim 50 \mathrm{fs}, \sim 3 \mathrm{~nJ}$ per pulse, $108 \mathrm{MHz}$ repetition rate) pulses. These femtosecond pulses are subsequently amplified by the Nd:YLF laser in an optical parametric amplifier (OPA). As a result $110 \mathrm{fs}$ (FWHM)near infra-red (NIR) laser pulses at a wavelength of $800 \mathrm{~nm}$ and a pulse energy of up to $30 \mu \mathrm{J}$ with the burst mode structure of the FEL can be produced. Either the $110 \mathrm{fs}$ infra-red pulses or the optical $12 \mathrm{ps}$ pulses can be sent to the experiments through a dedicated optical beam line system, see Fig. 7.

The relevant parameter for intensity dependent phenomena is the power density $I$ in units of $\mathrm{Wcm}^{-2}$. Hence, (i) the time behavior of the laser pulse and (ii) 
the spatial profile of the focussed laser are equally important for determining the intensity over the interaction region. The pulse duration of the amplified laser pulse is monitored using a single shot autocorrelator. A typical correlation trace shown in Fig. 5 shows a correlation width of about $150 \mathrm{fs}$ which corresponds to an actual pulse duration of about 110 fs FWHM (assuming a Gaussian shape).

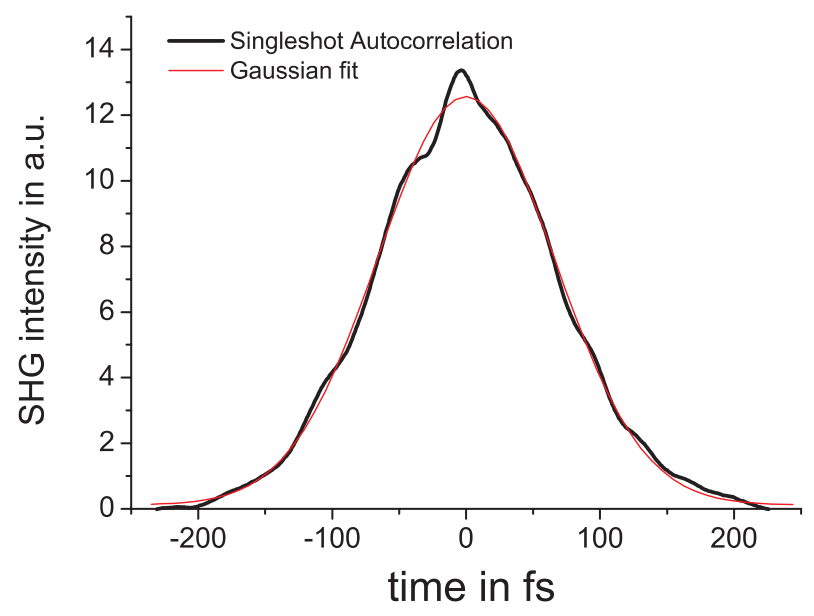

Fig. 5. Single-shot autocorrelation of the amplified laser pulse. The Gaussian fit to the measured curve has $150 \mathrm{fs}$ FWHM which corresponds to a pulse duration of 110 fs FWHM.

The optical laser was focussed via an off-axis parabola $\left(30^{\circ}\right.$ off-axis, $f=$ $272 \mathrm{~mm}, 76 \mathrm{~mm}$ diameter). In order to realize the collinear interaction geometry of FEL and optical laser, the parabola has a centered hole of $6 \mathrm{~mm}$ diameter to allow the FEL to pass (see Fig. 7). The results of simulations for the optical path do not show any significant degradation of the beam profile. However, in the experiment, slight deviations from the parabolic shape near the hole, created wings in the focal spot shape, see Fig. 6.

Measurements of the beam profile at the interaction region were obtained imaging the laser intensity distribution in the focal region onto a synchronized CCD camera. A $15 \mathrm{~mm}$ focal length lens was used to magnify the laser focal 
spot 20 times. The image allowed the determination of the focal spot shape and size. Fig. 6 shows a lineout of the focal region. The central part of the measured beam profile can be well described by a Gaussian profile of $15 \mu \mathrm{m}$ FWHM, but the outer wings are clearly affected by the diffraction effects from the mirror hole in the parabola. Despite this beam degradation, the focussing system for the optical laser still permits peak intensities of up to $10^{13} \mathrm{~W} / \mathrm{cm}^{2}$ to be obtained.

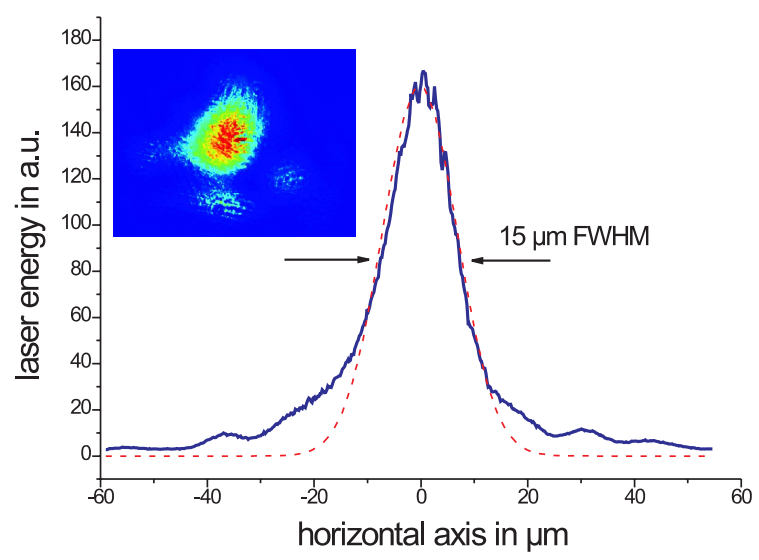

Fig. 6. Lineout of the $800 \mathrm{~nm}$ beam profile at the focus imaged at a magnification of 20 onto the CCD camera. The dashed line shows a Gaussian profile with a FWHM of $15 \mu \mathrm{m}$.

\section{TWO-COLOR EXPERIMENTAL SETUP}

\subsection{Magnetic bottle electron spectrometer}

Measurements of the kinetic energy of the photoelectrons were made by using a magnetic bottle electron spectrometer (MBES) [10,23]. An overview of the experimental arrangement is given in Fig. 7. Electrons ejected after the interaction of an effusive gas jet with the EUV beam in the acceptance volume of the MBES are directed by a strong permanent magnet $(0.5 \mathrm{~T})$ towards a $65 \mathrm{~cm}$ 
long flight tube. A solenoid producing a weak guiding field $(0.5 \mathrm{mT})$ brings almost all the electrons to a microchannel plate detector (MCP). A time-offlight (TOF) detection scheme allows the kinetic energies of all photoelectrons to be measured simultaneously. Hence, the MBES setup can be applied to low density targets and, in the case of the intense FEL beam, allows an analysis of the photoionization process on a shot-to-shot basis $[11,15]$. The best energy resolution of $1-2 \%$ of the kinetic energy was achieved by decelerating the electrons with the aid of electrostatic retardation fields at the entrance of the flight tube. All signals from the MCP detector were first read by the digital oscilloscope (LeCroy Wavemaster 8600A) where they were either averaged or the single-shot spectra were acquired at the macropulse repetition rate of $2-$ $5 \mathrm{~Hz}$.

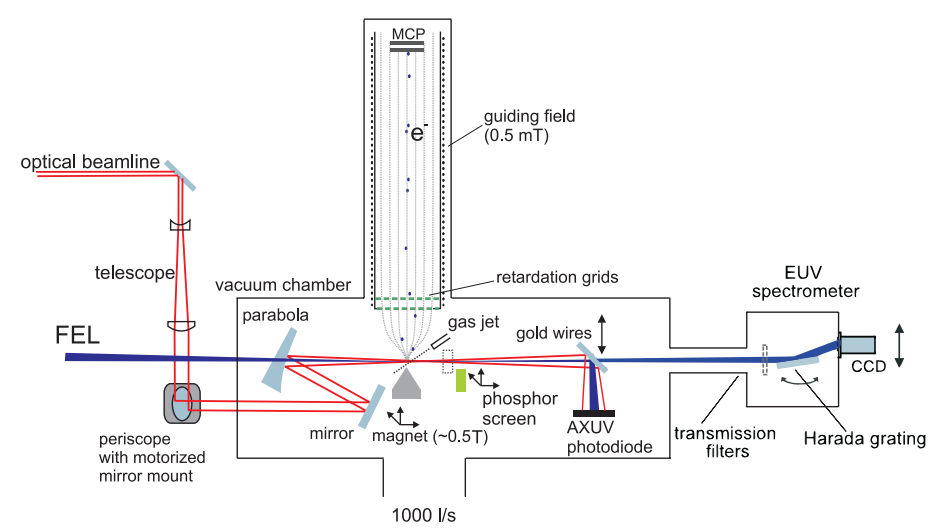

Fig. 7. Experimental setup for the pump-probe experiments using the combination of the FEL with an optical laser.

\subsection{Effects of space charge}

It is important to maintain a clean vacuum chamber in two-color photoionization experiments because the high intensities and short wavelengths of the EUV beam are sufficient to ionize most vacuum chamber contaminants. 
The interaction region in this experiment was housed in a vacuum chamber maintained by a turbomolecular pump capable of reaching a base pressure of $5 \times 10^{-10}$ mbar after baking. The rare gas pressure in the chamber was varied from $2 \times 10^{-5}$ to $5 \times 10^{-8}$ mbar by changing the rate of flow through a leak valve.

There are several factors which dictated the rare gas density used in this experiment. (i) The MCP detector requires a pressure which is lower than $1 \times 10^{-4}$ mbar. (ii) The presence of the gas of interest (usually a rare gas) should be significantly higher than the base pressure of the chamber. (iii) The significantly increased charge flux, produced by the intense EUV FEL, which then falls on the microchannel plate, induces saturation effects in the photoelectron signal. (iv) Space charge distorts the electron energies if there is too much ionization.

Except at very low FEL intensities, the last item is by far the most important. In general, experiments at FLASH which are sensitive to electron energies, spectral widths, and total yields, distortion by space charge is an undesirable effect. Here, the use of a tight focussing geometry and minimum gas densities helped to reduce the these effects. The volume of the FEL at the focus of the PG2 monochromator beamline was approximately $10^{-5} \mathrm{~cm}^{-3}\left(\sim 100 \mu \mathrm{m}^{2}\right.$ FEL focus $\times 1 \mathrm{~mm}$ MBES acceptance length). At a pressure of $5 \times 10^{-6} \mathrm{mbar}$ the number of rare gas atoms in this volume is approximately $10^{6}$. During the interaction with the FEL pulse the ions are essentially static, hence, as the fast photoelectrons leave the interaction volume the remaining electrons see an increasingly attractive potential. For $10^{5}$ singly charged ions in a spherical region of $10^{-5} \mathrm{~cm}^{-3}$, an electron on the surface sees a potential of the order of one electronvolt. 


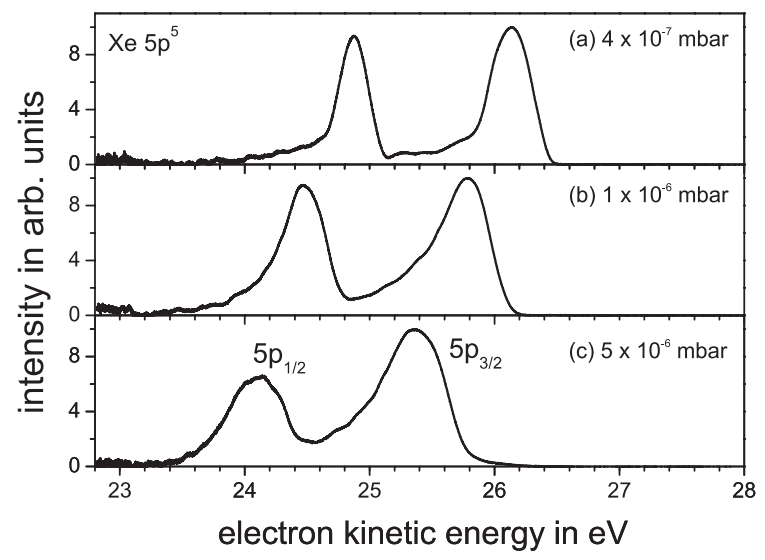

Fig. 8. Photoelectron spectra of xenon recorded after interaction with EUV photons from the FEL lasing at $32.4 \mathrm{~nm}(38.3 \mathrm{eV})$ at the first order of the PG2 beamline. The energy spectra demonstrate the space charge induced broadening of the $5 p$ photolines, (a) corresponds to xenon pressure of $4 \times 10^{-7} \mathrm{mbar}$, (b) is for $1 \times$ $10^{-6} \mathrm{mbar}$, and (c) is for $5 \times 10^{-6} \mathrm{mbar}$. 
The effect of space charge on the xenon photoelectron spectrum is shown in Fig. 8 for ionization of the 5p electronic subshell (binding energies are $12.1 \mathrm{eV}$ and $13.4 \mathrm{eV}$ for the spin-orbit components $5 \mathrm{p}^{-1}{ }^{2} \mathrm{P}_{3 / 2}$ and ${ }^{2} \mathrm{P}_{3 / 2}$, respectively.) These spectra were recorded at three different xenon densities using the first order of the monochromator at PG2 $\left(\hbar \omega_{F E L}=38.3 \mathrm{eV}\right)$. We found that space charge manifests itself through an asymmetric broadening, and shift of the photoelectron peaks up to $0.8 \mathrm{eV}$. Space charge effects were kept to minimum by changing the rare gas pressure in accord with the changes in the FEL pulse energy. At sufficiently high pulse energies and gas pressures, which result in a large effective species density for ionization, the space charge becomes sufficiently large to interfere with accurate measurements of the two-color sideband signal. These effects set the upper limit on the gas pressure used in the two-color data reported here to a few $10^{-7}$ mbars. In addition, the criterion for minimizing space charge effects reduced at the same time the occurrence of saturation of the MCP detector in the time-of-flight spectrometer.

\subsection{Single-shot mode}

Figure 9 shows a series of single-shot photoelectron spectra for one microbunch. In this case we used the first order of the PG2 monochromator beamline to select the third harmonic $3 \omega(114.9 \mathrm{eV})$ of the FLASH fundamental $\left(\hbar \omega_{F E L}\right.$ $=38.3 \mathrm{eV}$ ) for photoionization. Maximum resolution was obtained by applying retardation fields as well as closing the exit slit of the monochromator to $300 \mu \mathrm{m}$. The main peak at an electron kinetic energy of $45-47 \mathrm{eV}$ results from the $\mathrm{Xe} 4 \mathrm{~d}$ photolines (binding energies of $67.5 \mathrm{eV}$ and $69.5 \mathrm{eV}$ ) for the two $4 \mathrm{~d}^{-1}$ spin-orbit components, respectively. The broad feature at kinetic 
energies around $100 \mathrm{eV}$ is caused by the now unresolved 5p lines and some intensity due to the ionization of the 5s subshell (binding energy of $23.1 \mathrm{eV}$ ). The normal 4d-Auger transitions can be identified in the low kinetic energy region $(25-35 \mathrm{eV})$. These lines are resolved with much higher resolution, since their width is independent from the spectral width of the exciting FEL radiation. As for the single-shot spectra for the spectral distribution of the FLASH pulses (Fig. 2), the electron spectra show strong intensity variations when comparing the individual spectra. This is again related to fluctuation in the FEL pulses and is even enhanced in the third harmonic, since its intensity is proportional to the cube of that of the fundamental [15].

The average energy of FLASH was $70 \mu \mathrm{J}$, i.e. near or at saturation, having $0.5-1 \%$ in $3 \omega$ as discussed in Ref. [15]. From Ref. [18] the transmission of the PG2 beamline at a photon energy of $114.9 \mathrm{eV}$, a constant fixed focus value of 1.5 , and an exit slit of $300 \mu \mathrm{m}$ is estimated to be approximately $4 \%$. Therefore, overall we can expect approximately $30 \mathrm{~nJ}$ FEL beam energy at the experiment. Thus Fig. 9 demonstrates quite clearly the usefulness of the high sensitivity of the MBES setup in exploiting the high harmonic properties of FLASH at saturation. With an upgrade of the electron beam accelerator already underway, the shortest fundamental wavelength of $6.4 \mathrm{~nm}(190 \mathrm{eV})$ is expected. Hence, the possibility to make single-shot use of the unique harmonic structure of FLASH should be of great interest to the gas phase community.

\subsection{Spatial overlap between EUV and optical laser}

Special care has to be taken to ensure the best spatial overlap of the focal spots of the FEL generated EUV radiation and the optical laser beam. Using 


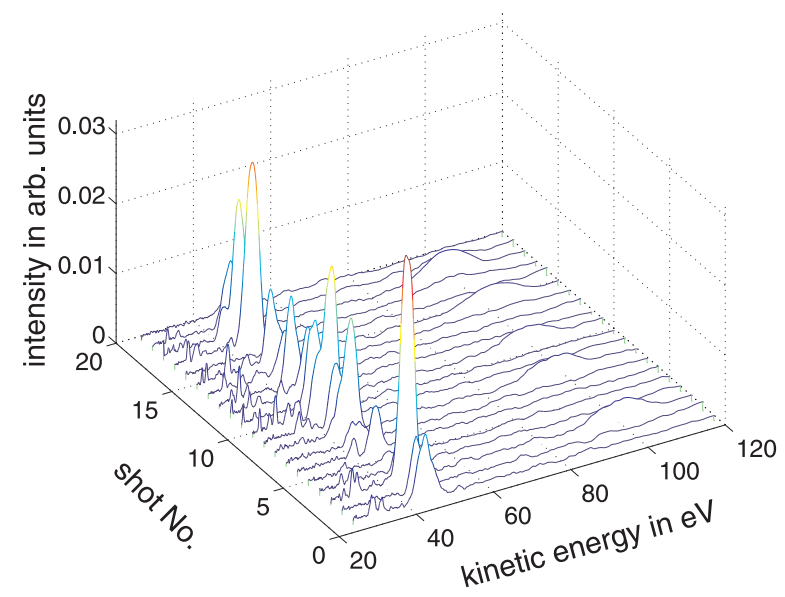

Fig. 9. Single-shot electron photoelectron spectra from xenon after interaction with EUV photons from the third harmonic $(\sim 10.8 \mathrm{~nm}, 114.9 \mathrm{eV})$ of the FEL lasing at $32.3 \mathrm{~nm}(38.3 \mathrm{eV})$ for one microbunch. The main peak at about $45 \mathrm{eV}$ results from photoemission from the unresolved Xe $4 d$ photolines. 
a motorized UHV manipulator a $10 \times 10 \mathrm{~mm}$ screen was moved into the interaction region. The screen was coated (by Proxitronic) with a $\sim 15 \mu$ m layer of $\mathrm{P} 46$ Phosphor $\left(\mathrm{Y}_{3} \mathrm{Al}_{5} \mathrm{O}_{12}\right.$ : Ce, $2.8 \mu \mathrm{m}$ average grain size). The phosphor fluoresces in the visible range (green) when illuminated with the EUV radiation of the FEL while the small grain size acts as a scattering screen for the optical laser. Substantial blurring of the phosphorescent spot was prevented by the small phosphor grain size and layer thickness.

The images of each spot were monitored with a long working distance $(34 \mathrm{~cm})$ microscope with 1.5 times magnification. Due to the small numerical aperture of the optical setup the diffraction limited resolution is around $7 \mu \mathrm{m}$ which is sufficient to overlap the focal spots. On the CCD camera that acquired the images 1 pixel corresponds to $4.3 \mu \mathrm{m}$. A typical image of the FEL focus is shown in Fig. 10. The recorded focal spot of $\sim 30 \mu \mathrm{m}$ is consistent with previous measurements ( e.g. [16]). In order to prevent damaging of the thin phosphor layer the FEL as well as the optical laser had to be substantially attenuated.

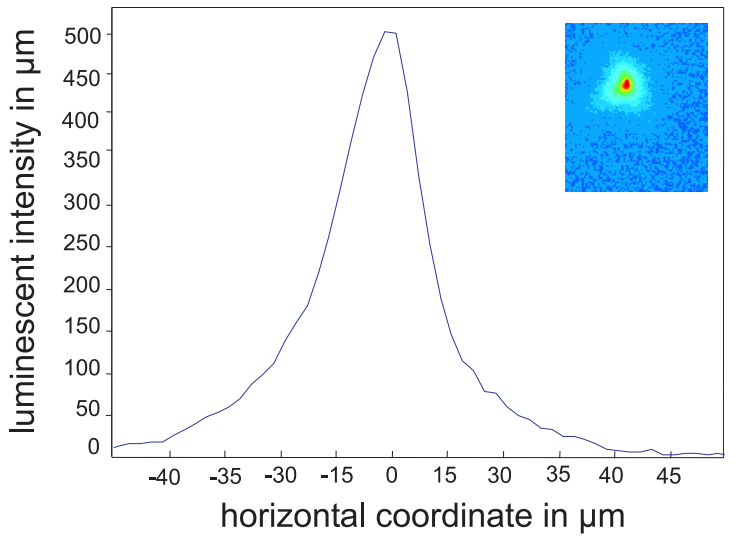

Fig. 10. Spatial profile of the FLASH beam. The inset shows the image of the FEL on a luminescent screen monitored with a long distance microscope.

Since the FEL focal spot position cannot be easily altered the optical laser 
had to be moved in all 3 spatial coordinates to overlap the FEL focal spot. The focal spot was moved in a plane normal to the incident laser beam by changing the direction of the beam slightly. The last mirror mount outside the vacuum chamber was equipped with stepper motors. By tilting the beam \pm $1.5 \mathrm{mrad}$ the focal spot could be moved by $\pm 500 \mu \mathrm{m}$ in the focal plane. Due to the astigmatism of the parabola the spot size increases slightly and the peak intensity decreases by less than $10 \%$.

In order to vary the longitudinal position of the focal spot the divergence of the beam was altered. A 1:1.5 Galilean telescope $\left(f_{1}=-200 \mathrm{~mm}\right.$ and $f_{2}=$ $300 \mathrm{~mm}$ ) was utilized to move the focus by $\pm 1 \mathrm{~mm}$ along the propagation direction without decreasing the intensity by more than $10 \%$. The second lens is mounted on a motorized translational stage. By changing the distance between the two lenses away from the nominal telescope distance the divergence is changed resulting in different longitudinal focal positions.

\subsection{Temporal overlap between EUV and optical pulses}

Full technical details of the synchronization between the FEL and the optical fs laser will be presented elsewhere [21] and so what follows is limited to salient operational details. The optical laser is synchronized to the radio frequency $(\mathrm{RF})$ source providing $1.3 \mathrm{GHz}, 108 \mathrm{MHz}$ and $9 \mathrm{MHz}$ driving the electron accelerator. These reference frequencies are delivered by a $300 \mathrm{~m}$ long temperature stabilized cable into the laser hutch. The $12^{\text {th }}$ harmonic of the repetition rate of the fs-laser $(1.3 \mathrm{GHz})$ is continuously compared to the reference frequency and if deviations are detected, control electronics adapt the repetition rate of the laser by changing the cavity length with a piezo. Cur- 
rently the synchronization to the $\mathrm{RF}$ is better than $150 \mathrm{fs}$ r.m.s. in an interval from $\mathrm{kHz}$ to several minutes.

Once the time delay between the EUV and optical pulses has been fixed, deviations of this value are monitored by a streak camera (C5680 by Hamamatsu) situated in the laser hutch, see Fig. 1. Less than $10^{-6}$ of the optical laser light used for the experiments is transported to the Streak camera as markers for the arrival time of the laser pulse. Secondly, a reference for the arrival time of the FEL is required. Here, we use a flash of light which is produced while the electrons, after passing the undulator, are bent via a dipole magnet into the electron dump at the end of the accelerator (called dipole radiation). This pulse, in which all optical wavelengths are present, is as short as the electron bunch (below $100 \mathrm{fs}$ ) and is inherently synchronized to the EUV pulse. The dipole radiation is guided into the laser hutch by a separate $55 \mathrm{~m}$ long beamline and is focused on the slit of the Streak camera. Thus, the measurement is parasitic and independent of the actual experiment. Due to the dispersion of the white dipole radiation pulse in the focussing lens of the streak camera, the pulse broadens tremendously to about 10 ps. Fig. 11 (a) shows both pulses on the streak camera.

The important information is the relative jitter between both pulses. In order to extract this information for each pair of pulses, the data are fitted to a Gaussian function and the difference between the peak position of the two pulses is used for further investigation. Fig. 11 (b) represents an 11 hour section of a timing stability measurement. The plot shows that the relative jitter of the two independent sources is only about $800 \mathrm{fs}$ over the entire $11 \mathrm{~h}$ period. A large contribution of the measured jitter results from the analysis of the data itself, however, calibration measurements showed that the peak 


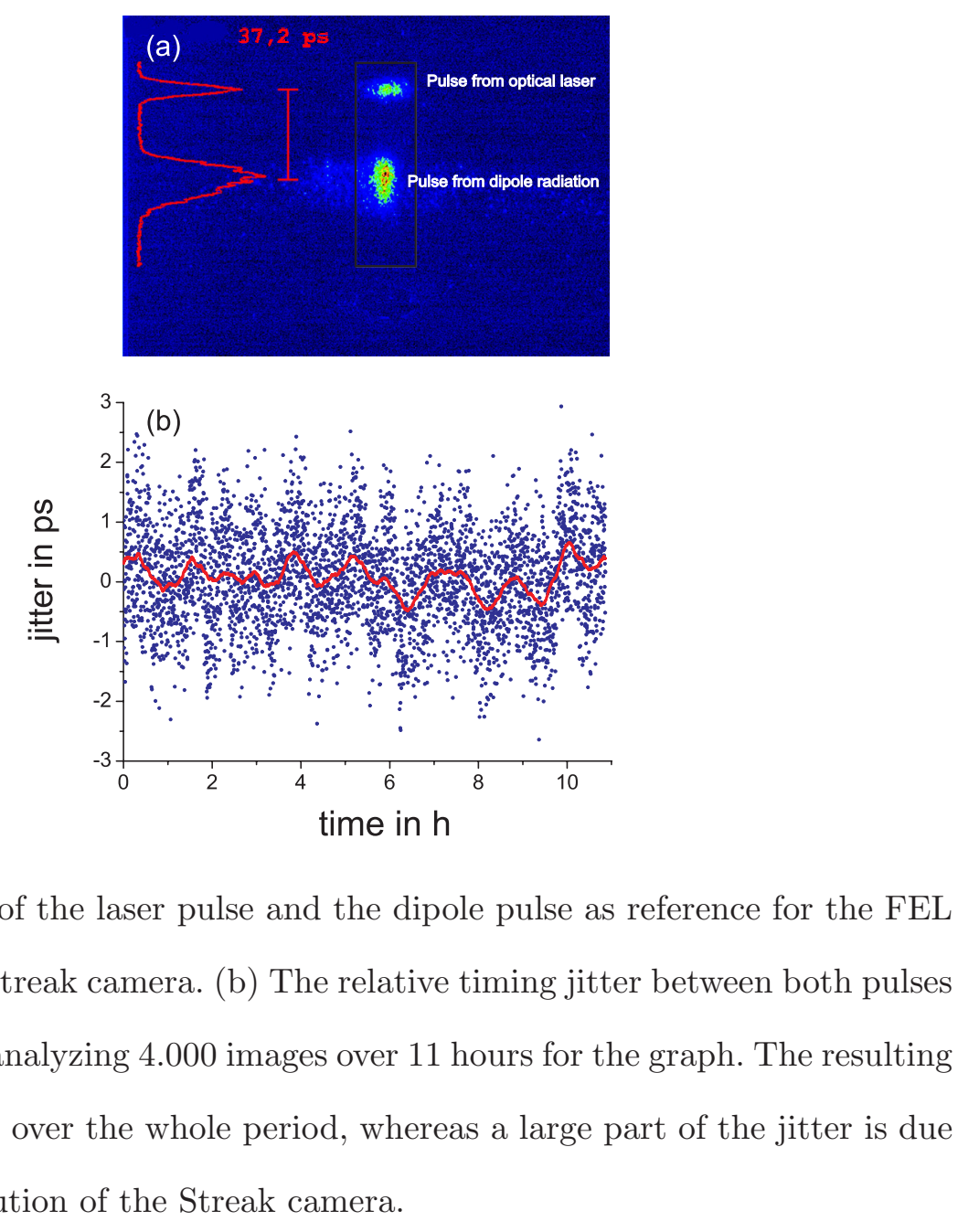


position can be determined with a resolution of about $400 \mathrm{fs}$ rms. That is, by analyzing only the center position, the measured resolution (400 fs) is well below the nominal 2 ps resolution of the Streak camera - the response function of the streak camera to a delta pulse.

\subsection{Temporal overlap within the experimental chamber}

The streak camera is necessary to monitor the drifts in the relative time delay between the EUV and NIR pulses, which is done parasitically and is independent of the actual experiment. However, an important issue for pump-probe experiments performed with two independent (however well synchronized) sources is the detection of the temporal overlap in the experimental chamber, which at FLASH is on average $70 \mathrm{~m}$ from the exit aperture of the FEL and up to $20 \mathrm{~m}$ from the optical laser. Hence finding the temporal separation between the EUV and NIR pulses at the point of interaction on a femtosecond timescale by scanning the optical time delay and monitoring sideband intensities is not a very practical proposition.

In order to vary the temporal delay between the optical laser and the FEL a computer controlled optical delay line housed in the laser hutch can be utilized. This delay line allows changes of up to 3 ns whereas larger delays can be achieved by electronically shifting the laser pulses up to several microseconds with respect to the FEL. A means of coarse tuning is needed before this fine tuning method can be usefully employed. The solution is a fast photodiode which views a fraction of the light scattered from the overlapped beams by a gold wire, see Fig. 7. 


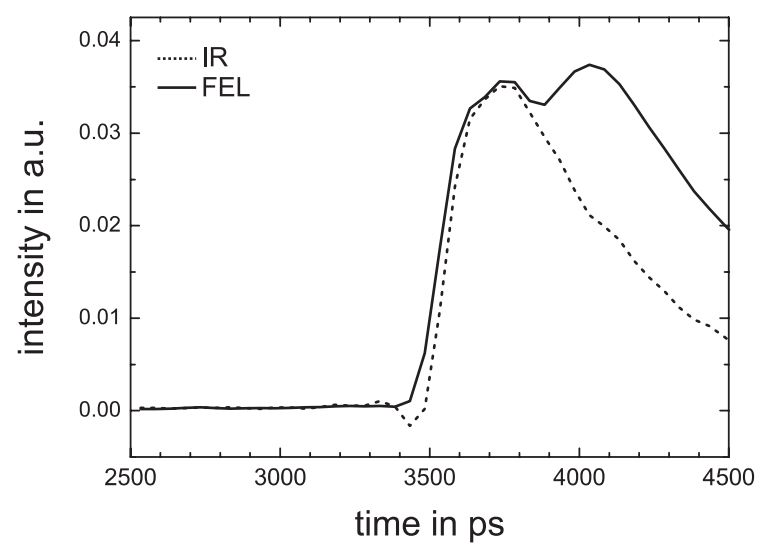

Fig. 12. Typical traces on the oscilloscope showing the response of the fast photodiode to the FLASH and NIR laser pulses, respectively. 
We chose the AXUV HS series from International Radiation Detectors Inc. for a number of reasons. Firstly, these diodes possess fast rise times ranging from 50 ps (HS6) to 200 ps (HS4), they are sensitive over a broad photon energy range from the NIR to the EUV so that both pulses could be viewed on a single diode and hence oscilloscope channel while finally, and importantly for our application, they are radiation hardened. We settled on the HS3 diode as the best compromise between sensitivity and rise time ( $80 \mathrm{ps})$. To maintain this high speed performance we used a fast bias-T, Model 5541A from Picosecond Pulses Labs of Boulder Co.

Typical traces showing the FEL and optical laser are shown in Figure 12. These traces were obtained with the aid of a LeCroy Wavemaster 8600A (20 GS/s) oscilloscope for signal capture. As the two pulses are collinear the traces were distinguished by closing the shutters alternatively in each beam. The time delay between pulses was determined by measuring the separation between the risetimes of each trace. The system permitted a shift of \pm 25 ps to be readily detected. Hence we were able to reduce the fine-tuning window to this range which corresponds to a spatial scanning range of ca. $\pm 10 \mathrm{~mm}$. The ultimate time resolution of $<1$ ps was found by scanning the delay stage through the $\pm 25 \mathrm{ps}$ and searching for evidence of two color above threshold ionization, discussed in detail below.

\section{TWO-COLOR TWO-PHOTON IONIZATION OF RARE GASES}

The most accurate method to obtain the relative time delay on a time scale comparable with the FEL and optical laser pulse widths (i.e., on the femtosecond scale) is the two color above threshold ionization technique discussed 
earlier. In this case, when the EUV and NIR pulses are overlapped in space and time, photoelectrons generated by the EUV pulse are born into the intense field of the optical laser where they can absorb or emit one or more NIR photons resulting in sidebands in the corresponding photoelectron spectrum of that target atom $[10,11]$.

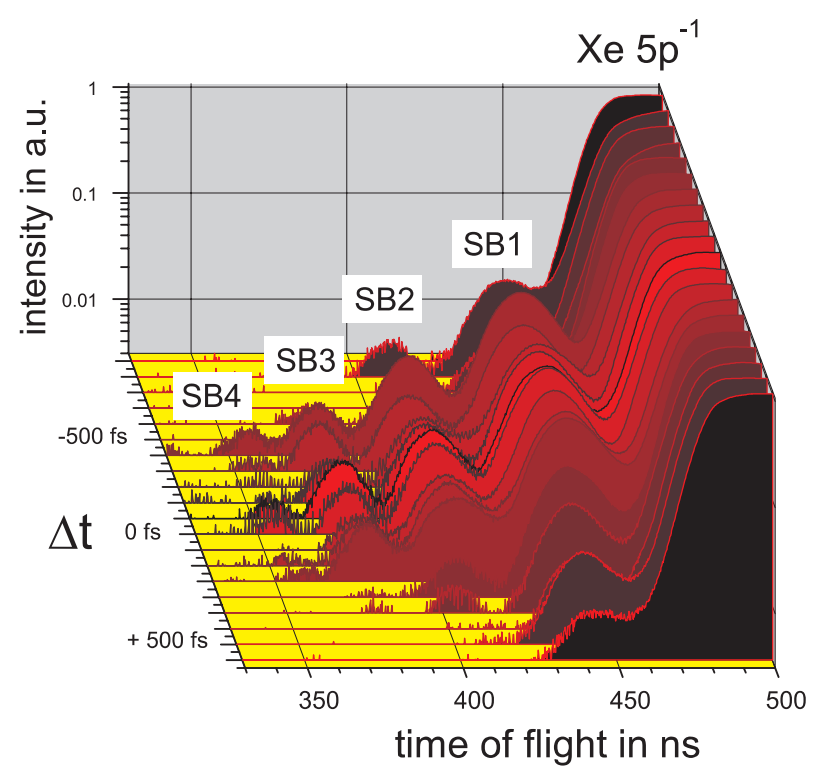

Fig. 13. Part of the time-of-flight spectrum for the two-photon ionization of xenon atoms by the EUV radiation from the FEL. Different spectra are given as a function of the time delay between the FLASH pulses ( $20 \mathrm{fs}, 13.8 \mathrm{~nm}$ ) and the optical (120 fs, $800 \mathrm{~nm}, 20 \mu \mathrm{J})$ laser.

In order to obtain fine control over the temporal delay between the optical laser and the FEL a computer controlled optical delay line, housed in the laser hutch, is used. The synchronization of the EUV and NIR pulses via a two-color pump-probe scheme is demonstrated using xenon as the target gas. The reader is referred to $[10,11]$ for full details of the technique.

The two-color ATI signal of the 5p photoionization of xenon atoms was measured at the micro focus beamline BL2 at a FEL fundamental wavelength of $13.8 \mathrm{~nm}\left(\hbar \omega_{F E L}=89.9 \mathrm{eV}\right)$, see Fig. 13. Each time-of-flight spectrum is the 
averaged result of 250 free electron + optical laser shots. The high field of the NIR laser gives rise to pronounced sideband structure, in particular higher sidebands (SB1 - 4) caused by the absorption of up to four optical photons are observed. Furthermore, the sidebands are observed only in a temporal region of $\sim 600 \mathrm{fs}$.

The width of the FEL + NIR overlap ( 600 fs FWHM), as obtained from the sideband yield as a function of the delay, is mainly determined by the temporal jitter of FEL pulses with respect to the optical laser and represents an overall temporal resolution of $\sim 250 \mathrm{fs}$ r.m.s., see [11] for further details. This resolution can be obtained for any pump-probe experiment at FLASH without further characterization of the relative temporal delay between both pulses.

In order to go beyond this temporal resolution imposed by the jitter of the FEL, we have also recorded single-shot spectra. In Fig. 14 some selected singleshot two-color Xe photoionization spectra, recorded at a FEL fundamental wavelength of $13.8 \mathrm{~nm}(89.9 \mathrm{eV})$, at the nominal maximum of the temporal overlap $\Delta \mathrm{t}=0$ are displayed showing again the $5 \mathrm{p}$ photolines and the high kinetic energy sidebands. The strongest sideband intensity, i.e. Fig. 14 (a), is only obtained for near $100 \%$ overlap between both pulses. Here the sideband intensity becomes even stronger than that of the 5p main lines and multiphoton (4 NIR photons) processes become possible. In contrast, the absence of the sideband signal, Fig. 14 (d), indicates a time difference larger than the width of the optical laser pulse. The comparison with a theoretical analysis of the sideband intensities [11] allow the temporal delay between both pulses to be determined to an accuracy of \pm 50 fs, i.e. $\Delta \mathrm{t}=(80 \pm 50) \mathrm{fs}$ and $(115$ $\pm 50)$ fs for Figs. 14 (b) and (c) respectively. In addition, the possibility to 


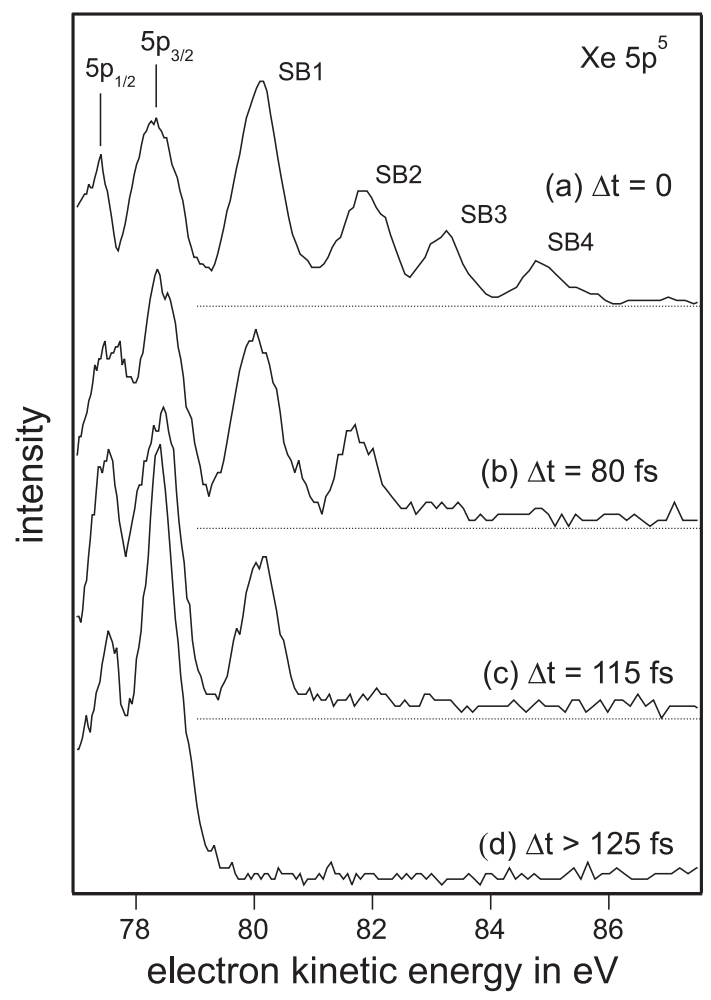

Fig. 14. (a) - (d) Single-shot spectra of xenon and the high-energy sidebands (SB1 - SB2) taken near the focus of BL2 $\left(\sim 100 \mu \mathrm{m}, \hbar \omega_{F E L}=89.9 \mathrm{eV}\right)$. (a) Maximum temporal overlap $\Delta \mathrm{t}=0$ produces 4 sidebands. (b) - (d) spectra at $\Delta \mathrm{t} \neq 0$ due to the jitter of the FEL pulse with respect to the NIR dressing pulse, which shows up as varying sideband intensity. 
record single shot spectra enable is to select only those spectra, which belong to a certain time delay, e.g. $\Delta t=0$. This increases tremendously the relative intensity of the interesting two-photon features as well as the efficiency for various pump-probe experiments, despite the temporal jitter between the two pulses.

\section{SUMMARY}

In the present paper, we have outlined the salient experimental considerations underpinning successful two-color pump-probe experiments which use EUV FEL $(10-50 \mathrm{fs})$ pulses from FLASH in combination with a synchronized optical fs (110 fs) laser. A number of specific methodologies and techniques used to characterize and control the FEL and the optical laser pulses are discussed. Particular attention is given to the procedures employed to optimize the spatial and temporal overlap of both pulses. In order to fully explore and exploit the properties of the FLASH pulses, the necessity for single-shot characterization has been demonstrated. To both showcase the two-color pump-probe setup and determine limits on its applicability and stability, an experiment on above threshold ionization (ATI) in rare gases has been performed. Averaging over many thousands of pulses, a temporal resolution of $250 \mathrm{fs}$ r.m.s. was determined. This value is mainly determined by the jitter between the pulses of the FEL and the optical laser. Using single-shot analysis, the delay time can be determined with greater accuracy and a temporal resolution of at least $50 \mathrm{fs}$ can be obtained. The methods described here for applications in the gas phase can be directly translated to pump-probe studies on other targets such as plasmas or solids. For the future, the control and complete characteriza- 
tion of each individual FEL and optical pulse will be a prerequisite for the successful realization of many unique ultrafast time-resolved investigations in the EUV regime.

\section{Acknowledgements}

We thank the scientific and technical team at FLASH, in particular E. Saldin, E. A. Schneidmiller and M. Yurkov as well as the machine operators and run coordinators, being the foundation of the successful operation and delivery of the EUV-FEL beam. Support from the EU RTD-project X- Ray FEL PumpProbe HRPI-CT-1999-50009 is acknowledged. Support from Science Foundation Ireland - Frontiers (DCU researchers), Ulysses France-Ireland (DCU and LIXAM researchers) and Higher Education Authority - North-South (DCU and QUB researchers) schemes are also acknowledged.

\section{References}

[1] V. Ayvazyan et al., Eur. Phys. J. D. 37297 (2006).

[2] W. Ackermann et al., Nature Photonics 1, 336 (2007).

[3] F. J. Wuilleumier and M. Meyer, J. Phys. B 39, R425 (2006)

[4] C. G. Ng, J. Electron Spectrosc. Rel. Phenom. 142, 179 (2005)

[5] P. Wang, H. K. Woo, K. C. Lau, X. Xing, C. Y. Ng, A. S. Zyubin, and A. M. Mebel, J. Chem. Phys. 124, 064310 (2006)

[6] X. Xing, B. Reed, K.-C. Lau, C. S. Lam, and C. Y. Ng, J. Chem. Phys. 127, $044313(2007)$ 
[7] J. M. Schins, P. Breger, P. Agostini, R. C. Constantinescu, H. G. Muller, G. Grillon, A. Antonetti, and A. Mysyrowicz, Phys. Rev. Lett. 73, 2180 (1994).

[8] T. E. Glover, R. W. Schoenlein, A. H. Chin, and C. V. Shank, Phys. Rev. Lett. 76, 2468 (1996).

[9] E. S. Toma, H. G. Muller, P. M. Paul, P. Breger, P. Agostini, C. Le Blanc, G. Mullot, and G. Cheriaux, Phys. Rev. A 62, 061801(R) (2000).

[10] M. Meyer, D. Cubaynes, P. O'Keeffe, H. Luna, P. Yeates, E. T. Kennedy, J. T. Costello, P. Orr, R. Taïeb, A. Maquet, S. Düsterer, P. Radcliffe, H. Redlin, A. Azima, E. Plönjes, and J. Feldhaus, Phys. Rev. A. 74, 011401(R) (2006).

[11] P. Radcliffe, S. Düsterer, A. Azima, H. Redlin, J. Feldhaus, J. Dardis, K. Kavanagh, H. Luna, J. Pedregosa Gutierrez, P. Yeates, E. T. Kennedy, J. T. Costello, A. Delserieys, C. L. S. Lewis, R. Taïeb, A. Maquet, D. Cubaynes and M. Meyer, Appl. Phys. Lett. 90, 131108 (2007).

[12] R. Treusch, Ch. Gerth, T. Lokajczyk, J. Feldhaus, Nucl. Instrum. and Methods A 467-468 (Part 1), 30 (2001), and Ch. Gerth, B. Faatz, T. Lokajczyk, R. Treusch, J. Feldhaus, Nucl. Instrum. and Methods A 475 (Part 1), 481.

[13] K. Tiedtke in HASYLAB Annual Report 2003, Section "Technical Developments at HASYLAB", subsection "VUV-FEL" and K. Tiedtke, J. Feldhaus, Ch. Gerth, U. Hahn, U. Jastrow, E. Plönjes, B. Steeg and R. Treusch, AIP Conf. Proc. 705, 572-575 (2004).

[14] R. Treusch in HASYLAB Annual Report 2005, Section "VUV-FEL", subsection "Photon Diagnostics for the VUV-FEL".

[15] S. Düsterer, P. Radcliffe, G. Geloni, U. Jastrow, M. Kuhlmann, E. Plönjes, K. Tiedtke, R. Treusch, J. Feldhaus, P. Nicolosi, L. Poletto, P. Yeates, H. Luna, J. T. Costello, P. Orr, D. Cubaynes, and M. Meyer, Opt. Lett. 31, 1750 (2006). 
[16] A. A. Sorokin, S. V. Bobashev, J. Feldhaus, Ch. Gerth, A. Gottwald, U. Hahn, U. Kroth, M. Richter, L. A. Schmaenok, B. Steeg, K. Tiedtke and R. Treusch, AIP Conf. Proc. 705, 557-560 (2004) and M. Richter, A. Gottwald, U. Kroth, A. A. Sorokin, S. V. Bobashev, L. A. Shmaenok, J. Feldhaus, Ch. Gerth, B. Steeg, K. Tiedtke and R. Treusch, Appl. Phys. Lett. 83, 2970-2972 (2003).

[17] A. A. Sorokin, A. Gottwald, A. Hoehl, U. Kroth, H. Schppe, G. Ulm, M. Richter, S. V. Bobashev, I. V. Domracheva, D. N. Smirnov, K. Tiedtke, S. Düsterer, J. Feldhaus, U. Hahn, U. Jastrow, M. Kuhlmann, T. Nunez, E. Plönjes, and R. Treusch, Appl. Phys. Lett. 89, 221114 (2006).

[18] M. Martins, M. Wellhöfer, J. T. Hoeft, W. Wurth, J. Feldhaus, and R. Follath, Rev. Sci. Instr. 77, 115108 (2006).

[19] L. Poletto, L. Epulandi, P. Nicolosi, M. Pelizzo, P. Zambolin, J. Feldhaus, U. Jastrow, U. Hahn, E. Ploenjes and K. Tiedtke, SPIE Proc. Vol. 5534, Fourth Generation X-Ray Sources and Optics II, (2004) and P. Nicolosi, L. Poletto, M.-G. Pelizzo, L. Epulandi, P. Zambolin, J. Feldhaus, U. Jastrow, U. Hahn, E. Plönjes, K. Tiedtke, Journ. of Elect. Spect. Rel. Phen. 144-147, 1055-1058, (2004).

[20] T. Kita, T. Harada, N. Nakano and H. Kuroda, Appl. Opt. 22, 512 (1983).

[21] H. Redlin et al., to be published (2007).

[22] I. Will, G. Koss, I. Templin, Nucl. Instrum. and Methods A 541, 467 (2005).

[23] J. Eland, O. Vieuxmaire, T. Kinugawa, P. Lablanquie, R. Hall, and F. Penent, Phys. Rev. Lett. 90, 530031 (2003).

[24] P. O Shea, M. Kimmel, X. Gu, and R. Trebino, Opt. Lett. 26, 932 (2001). 